\title{
ON HYPO-ELASTIC ANALOGUES OF THE DILATANT DOUBLE-SLIDING MODEL
}

\author{
N. P. KRUYT \\ Department of Mechanical Engineering, University of Twente, Enschede, The Netherlands
}

\section{SUMMARY}

The relation between the hypo-elastic constitutive law and Mehrabadi and Cowin's dilatant double-sliding model for cohesionless granular materials is studied. Conditions that must be satisfied by hypo-elastic analogues of the double-sliding model are derived constructively, and a simple example is given.

\section{INTRODUCTION}

Constitutive relations for two-dimensional deformations of cohesionless granular materials are considered. The granular materials are assumed to be isotropic and rate-independent.

One of the current trends in modelling the behaviour of granular materials is to study constitutive relations of the hypo-elastic type. ${ }^{1-6}$ The general hypo-elastic constitutive relation, originally proposed by Truesdell, ${ }^{7}$ is a generalization of the theory of nonlinear elasticity and states that the stress rate is determined by the current stress and the rate of deformation. The stress rate depends linearly on the rate of deformation. Tokuoka ${ }^{8-10}$ showed that the theory of hypoelasticity and the classical theory of plasticity are similar. He demonstrated that hypo-elastic constitutive equations can possess a yield surface, and also derived the corresponding flow rule. A critical comparison of the theory of hypo-elasticity and the classical theory of plasticity was given by Mroz. ${ }^{11}$ Mehrabadi and Cowin ${ }^{12}$ proved the existence of hypo-elastic analogues of Spencer's double-sliding model ${ }^{13,14}$ and its generalization for dilatant materials. ${ }^{15}$ Spencer's model is a well-known model which is especially relevant for large, continued deformations of cohesionless granular materials.

Here a constructive derivation is given of the conditions that must be fulfilled by hypo-elastic analogues of the dilatant double-sliding model.

Tensile stresses are reckoned positive, as is the usual convention in continuum mechanics. Let $\mathbf{u}$ denote the velocity vector and $\mathbf{T}$ the stress tensor. The rate of deformation tensor $\mathbf{D}$ is defined by

$$
\mathbf{D}=\frac{1}{2}\left[(\nabla \mathbf{u})+(\nabla \mathbf{u})^{\mathrm{T}}\right]
$$

and the spin tensor $\mathbf{W}$ by

$$
\mathbf{W}=\frac{1}{2}\left[(\nabla \mathbf{u})-(\nabla \mathbf{u})^{\mathrm{T}}\right]
$$

The deviator of $\mathbf{D}$ is given by

$$
\mathbf{D}^{\prime}=\mathbf{D}-\frac{1}{2}(\operatorname{tr} \mathbf{D}) \mathbf{1}
$$


The deviator $\mathbf{T}^{\prime}$ of the stress tensor is defined similarly. The mean pressure $p$ is given by

$$
p=-\frac{1}{2} \operatorname{tr} \mathbf{T}
$$

and the invariant $\tau$ of the stress deviator by

$$
\tau^{2}=\frac{1}{2} \operatorname{tr}\left(\mathbf{T}^{\prime} \cdot \mathbf{T}^{\prime}\right)
$$

\section{THE HYPO-ELASTIC CONSTITUTIVE RELATION}

Truesdell ${ }^{7}$ proposed the hypo-elastic constitutive relation:

$$
\stackrel{\circ}{\mathbf{T}}=\mathbf{H}(\mathbf{T}): \mathbf{D}
$$

The fourth-order tensor $\mathbf{H}$ is called the hypo-elasticity tensor. In (6) $\mathbf{T}$ denotes the objective Jaumann stress rate, which is defined by

$$
\dot{\mathbf{T}}=\dot{\mathbf{T}}-\mathbf{W} \cdot \mathbf{T}+\mathbf{T} \cdot \mathbf{W}
$$

The superimposed dot denotes the material time derivative. Note that (6) is linear in the rate of deformation. Equation (6) is a generalization of the theory of nonlinear elasticity, where

$$
\text { stress increment }=\text { function }(\text { stress }) \times \text { strain increment }
$$

Because of the symmetry of the stress tensor and the rate of deformation tensor, the hypoelasticity tensor $\mathbf{H}$ must meet the conditions

$$
\mathbf{H}_{i j k l}=\mathbf{H}_{j i k l}=\mathbf{H}_{j i l k}
$$

\section{HYPO-ELASTICITY AND YIELD SURFACES}

Tokuoka $^{8-10}$ showed that the hypo-elastic constitutive equation (6) can exhibit yield-like behaviour. The hypo-elastic yield surface is defined by the stress states for which the hypoelasticity tensor $\mathbf{H}$ becomes singular; when this occurs there is no longer a unique relation between stress rate and rate of deformation, which means that the deformation becomes plastic.

In the theory of hypo-elasticity the stress can never lie on the yield surface, contrary to the classical theory of plasticity where plastic deformation occurs only on the yield surface. This means that the hypo-elastic yield surface acts as an asymptote in the stress space, beyond which the stresses are not feasible.

Examples of hypo-elastic constitutive relations that possess the von Mises and Tresca yield surface are given in References 9 and 10.

\section{THE DILATANT DOUBLE-SLIDING MODEL}

A well-known model for large, continued deformations of granular materials is Spencer's model. ${ }^{13,14}$ It adopts the Coulomb failure criterion and supposes that deformation occurs by a superposition of two simple shearing motions along the sliplines (where the critical ratio of shear to normal stress is attained). In addition to the shearing deformation, the material deforms by rotation of the slipline field. In Spencer's original formulation it was assumed that the material is incompressible. Mehrabadi and Cowin ${ }^{15}$ generalized the model to include the effect of dilatancy. The material satisfies the Coulomb yield criterion

$$
\tau=\mu p
$$


where $\mu=\sin \phi, \phi$ being the angle of internal friction. The kinematic behaviour is modelled by the equations

$$
\mathbf{T} \cdot \stackrel{\mathbf{T}}{-} \mathbf{\mathbf { T }} \cdot \mathbf{T}=2 h_{1}(\mathbf{T} \cdot \mathbf{D}-\mathbf{D} \cdot \mathbf{T})
$$

and

$$
\beta \operatorname{tr}\left(\mathbf{T}^{\prime} \cdot \mathbf{D}^{\prime}\right)=\tau \operatorname{tr}(\mathbf{D})
$$

where

$$
\beta=\sin v / \cos (\phi-v)
$$

and

$$
h_{1}=\tau(\beta \mu-1) /(\mu-\beta)
$$

The dilatancy angle is denoted by $v$.

For particular values of $v$ two older theories are recovered: ${ }^{15}$ when $v=0$ these equations reduce to Spencer's original model, ${ }^{13}$ and the Drucker-Prager model ${ }^{16}$ is obtained when $v=\phi$.

\section{HYPO-ELASTIC ANALOGUES OF THE DILATANT DOUBLE-SLIDING MODEL}

The constitutive relation (6), in two dimensions, has the representation:

$$
\stackrel{\circ}{\mathbf{T}}=\left[g_{1} \operatorname{tr} \mathbf{D}+g_{2} \operatorname{tr}\left(\mathbf{T}^{\prime} \cdot \mathbf{D}^{\prime}\right)\right] 1+\left[g_{4} \operatorname{tr} \mathbf{D}+g_{5} \operatorname{tr}\left(\mathbf{T}^{\prime} \cdot \mathbf{D}^{\prime}\right)\right] \mathbf{T}^{\prime}+2 h_{1} \mathbf{D}
$$

This result follows from a representation theorem ${ }^{17}$ for isotropic functions. The functions $g_{i}$ and $h_{1}$ depend only on the invariants $p$ and $\tau$ of the stress tensor. Note that, when $h_{1}$ is given by (14), (15) always fulfils the first kinematic equation of the double-sliding model (11).

The conditions that must be met by hypo-elastic analogues of the dilatant double-sliding model will now be derived from the following requirements: (i) the constitutive law (15) must possess the Coulomb yield surface (ii) this law must obey the consistency relation and (iii) the dilatancy relation (12) is valid for stress states near the yield surface.

First equation (15) will be inverted, giving $\mathbf{D}$ as a function of $\mathbf{T}$ and $\mathbf{T}^{\circ}$. From (15) we obtain:

$$
\operatorname{tr}\left(\dot{\mathbf{T}}^{\prime} \cdot \mathbf{T}^{\prime}\right)=2 \tau^{2} g_{4} \operatorname{tr} \mathbf{D}+\left(2 \tau^{2} g_{5}+2 h_{1}\right) \operatorname{tr}\left(\mathbf{T}^{\prime} \cdot \mathbf{D}^{\prime}\right)
$$

and

$$
-\dot{p}=\left(g_{1}+h_{1}\right) \operatorname{tr} \mathbf{D}+g_{2} \operatorname{tr}\left(\mathbf{T}^{\prime} \cdot \mathbf{D}^{\prime}\right)
$$

Inverting (16) and (17) gives

$$
\begin{gathered}
\operatorname{tr}(\mathbf{D})=\frac{g_{2} \operatorname{tr}\left(\dot{\mathbf{T}}^{\prime} \cdot \mathbf{T}^{\prime}\right)+\dot{p}\left(2 \tau^{2} g_{5}+2 h_{1}\right)}{2 \tau^{2} g_{2} g_{4}-\left(g_{1}+h_{1}\right)\left(2 \tau^{2} g_{5}+2 h_{1}\right)} \\
\operatorname{tr}\left(\mathbf{T}^{\prime} \cdot \mathbf{D}^{\prime}\right)=-\frac{\left(g_{1}+h_{1}\right) \operatorname{tr}\left(\mathbf{T}^{\prime} \cdot \mathbf{T}^{\prime}\right)+2 \tau^{2} g_{4} \dot{p}}{2 \tau^{2} g_{2} g_{4}-\left(g_{1}+h_{1}\right)\left(2 \tau^{2} g_{5}+2 h_{1}\right)}
\end{gathered}
$$

Substitution of (18) and (19) into (15) gives, after some algebra,

$$
\begin{aligned}
2 h_{1} \mathbf{D}= & \left\{\dot{\mathbf{T}}^{\prime}-\frac{1}{2 \tau^{2}} \operatorname{tr}\left(\hat{\mathbf{T}}^{\prime} \cdot \mathbf{T}^{\prime}\right) \mathbf{T}^{\prime}\right\}-\frac{1}{Y}\left[\left\{\frac{1}{2 \tau^{2}}\left(g_{1}+h_{1}\right) 2 h_{1} \operatorname{tr}\left(\dot{\mathbf{T}}^{\prime} \cdot \mathbf{T}^{\prime}\right)+2 g_{4} h_{1} \dot{p}\right\} \mathbf{T}^{\prime}\right. \\
& \left.+\left\{-g_{2} h_{1} \operatorname{tr}\left(\dot{\mathbf{T}}^{\prime} \cdot \mathbf{T}^{\prime}\right)+\left[g_{1}\left(2 \tau^{2} g_{5}+2 h_{1}\right)-2 \tau^{2} g_{2} g_{4}\right] \dot{p}\right\} \mathbf{1}\right]
\end{aligned}
$$


where $Y$ is defined by

$$
Y=2 \tau^{2} g_{2} g_{4}-\left(g_{1}+h_{1}\right)\left(2 \tau^{2} g_{5}+2 h_{1}\right)
$$

Equation (20) is the inverted form of (15). The relation between $\mathbf{D}, \mathbf{T}$ and $\mathbf{T}^{\circ}$ is singular when $Y=0$, so $Y$ is the hypo-elastic yield surface. The constitutive relation (15) has the yield function $\tau=\mu p$ if

$$
Y \rightarrow 0 \text { as } \tau \rightarrow \mu p
$$

In addition, we require that the consistency relation is fulfilled:

$$
(\tau \dot{-} \mu p) \rightarrow 0 \text { as } \tau \rightarrow \mu p
$$

From (16) and (17) it follows that $(\tau \dot{-} \mu p)$ is given by

$$
(\tau \dot{-} \mu p)=\left[\tau g_{4}+\mu\left(g_{1}+h_{1}\right)\right] \operatorname{tr}(\mathbf{D})+\left(\tau g_{5}+\frac{h_{1}}{\tau}+\mu g_{2}\right) \operatorname{tr}\left(\mathbf{T}^{\prime} \cdot \mathbf{D}^{\prime}\right)
$$

If

$$
g_{4} \rightarrow-\mu \frac{g_{1}+h_{1}}{\tau}
$$

and

$$
g_{2} \rightarrow \frac{-1}{\mu}\left(\tau g_{5}+\frac{h_{1}}{\tau}\right)
$$

as $\tau \rightarrow \mu p$, the consistency relation (23) is satisfied.

The conditions under which the dilatancy relation (12) of the double-sliding model is fulfilled will now be derived. From (18) and (19) we find that if

$$
g_{2} \rightarrow-\beta \frac{g_{1}+h_{1}}{\tau}
$$

and

$$
\tau^{2} g_{5}+h_{1} \rightarrow-\beta \tau g_{4}
$$

as $\tau \rightarrow \mu p$, then the third equation (12) of the double-sliding model is satisfied.

Recapitulating, we have deduced that hypo-elastic analogues of the dilatant double-sliding model should satisfy (14), (22) and (25)-(28). These conditions are all fulfilled if

$$
\begin{aligned}
& h_{1}=\tau(\beta \mu-1) /(\mu-\beta) \\
& g_{2} \rightarrow-\beta \frac{g_{1}+h_{1}}{\tau} \\
& g_{4} \rightarrow-\mu \frac{g_{1}+h_{1}}{\tau} \\
& g_{5} \rightarrow \frac{1}{\tau^{2}}\left[(\beta \mu-1) h_{1}+\beta \mu g_{1}\right]
\end{aligned}
$$

as $\tau \rightarrow \mu p$.

No restrictions need to be imposed on $g_{1}$. These conditions only are limits as the yield surface is approached. The remaining degrees of freedom in the choice of the functions $g_{i}$ can be used to model the initial deformation behaviour. 


\section{CONCLUDING REMARKS}

A constructive derivation has been given of the conditions that must be satisfied by hypo-elastic analogues of the dilatant double-sliding model. These conditions, (29)-(32), concern the asymptotic form, as the Coulomb yield surface is approached, of the coefficients in the general hypoelastic constitutive equation.

A simple example is:

$$
\begin{aligned}
& \frac{h_{1}}{\tau}=(\beta \mu-1) /(\mu-\beta) \\
& g_{1}=0 \\
& g_{2}=-\beta \frac{h_{1}}{\tau} \\
& g_{4}=-\mu \frac{h_{1}}{\tau} \\
& g_{5}=\frac{(\beta \mu-1)}{\mu p} \frac{h_{1}}{\tau}
\end{aligned}
$$

As a final remark, it is noted that the model can be refined by assuming that the angle of dilatancy, and hence $\beta$, depends on the density. In analogy with the critical state model, ${ }^{18}$ we then have $\beta \rightarrow 0$ as $\rho \rightarrow \rho_{\mathrm{c}}$, where $\rho_{\mathrm{c}}$ is the density corresponding to the critical state.

\section{ACKNOWLEDGEMENTS}

This work was performed mainly while the author was visiting McGill University, Montreal. The author would like to thank Professor S.B. Savage for very valuable discussions and encouragement. Financial support from the Netherlands Foundation for Technical Research (S.T.W.) is also gratefully acknowledged. The University of Twente (and especially Professor F. J. C. Rademacher) is thanked for cooperation and support.

\section{REFERENCES}

1. M. Romano, 'A continuum theory for granular media with a critical state', Arch. Mech., 26, 1011-1028 (1974).

2. R. O. Davis and G. Mullenger, 'A rate-type constitutive model for soils with a critical state', Int.j. numer. anal. methods Geomech., 2, 255-282 (1978).

3. R. O. Davis and G. Mullenger, 'Derived failure criteria for granular materials', Int. j. numer. anal. methods Geomech., 3, 279-283 (1979).

4. L. Dragusin, 'A hypo-elastic model for soils', Int. J. Eng. Sci., 19, 511-552 (1981).

5. G. Mullenger and R. O. Davis, 'A unified yield criterion for cohesionless granular materials', Int. j. numer. anal. methods Geomech., 5, 285-294 (1981).

6. G. Gudehus and D. Kolymbas, 'An approach to cohesionless materials and cylindrical bins', in Proc. 1st World Congress on Particle Technology, Part 3 (K. Leschonski, Ed.), Nürnberger Messe- und Ausstellungsgesellschaft, Nürnberg. 1986, pp. 33-48.

7. C. Truesdell, 'Hypo-elasticity', J. Ration. Mech. Anal., 4, 83-133, 1019-1020 (1955).

8. T. Tokuoka, 'Yield conditions and flow rules derived from hypo-elasticity', Arch. Ration. Mech. Anal., 42, 239-252 (1971).

9. T. Tokuoka, "Tresca-type plastic materials in the theory of hypo-elasticity: Mechanical constitutive equations and simple shear deformation', Int. J. Non-Linear Mech., 7, 609-620 (1972).

10. T. Tokuoka, 'Hypo-elasticity with internal variables and derived failure condition', Z. Angew. Math. Mech., 47, 107-111 (1977).

11. Z. Mroz, 'On hypo-elasticity and plasticity approaches to constitutive modelling of inelastic behaviour of soils', Int. $j$. numer. anal. methods Geomech., 4, 45-55 (1980). 
12. M. M. Mehrabadi and S. C. Cowin, 'Granular material flow rules derived from rate independent flow rules', in Proc. IUTAM Conf. on Deformation and Failure of Granular Materials (P. A. Vermeer and H. J. Luger, Eds.), Balkema, Rotterdam. 1982, pp. 139-144.

13. A. J. M. Spencer, 'A theory of the kinematics of ideal soils under plane strain conditions', J. Mech. Phys. Solids, 12, 337-351 (1964)

14. A. J. M. Spencer, 'Deformation of ideal granular materials', in Mechanics of Solids (H. G. Hopkins and M. J. Sewell, Eds.), Pergamon Press, Oxford. 1982, pp. 607-652.

15. M. M. Mehrabadi and S. C. Cowin, 'Initial planar deformation of dilatant granular materials', J. Mech. Phys. Solids, 26, 269-284 (1978).

16. D. C. Drucker and W. Prager, 'Soil mechanics and plastic analysis or limit design', Q. Appl. Math., 10, 157-165 (1952).

17. C. C. Wang, 'A new representation theorem for isotropic functions, Parts I and II', Arch. Ration. Mech. Anal., 36, 166-197, 198-223 (1970).

18. A. Schofield and C. P. Wroth, Critical State Soil Mechanics, McGraw-Hill, New York, 1968. 